\title{
Neuro-Ophthalmic and Clinical Characteristics of Brain Tumors in Children in Macau
}

\author{
In Sim $\mathrm{Ng}^{1}$, Jorge Sales Marques ${ }^{2 *}$ and $\mathrm{Ao} \mathrm{Hei}^{2}$ \\ ${ }^{1}$ Ophthalmology Department, Centro Hospitalar Conde S. Januário, Macau, China \\ ${ }^{2}$ Pediatric Department, Centro Hospitalar Conde S. Januário, Macau, China
}

*Corresponding Author: Jorge Sales Marques, Pediatric Department, Centro Hospitalar Conde S. Januário, Macau, China.

Received: September 27, 2019; Published: November 07, 2019

DOI: 10.31080/ASPE.2019.02.0179

\section{Abstract}

The objective of this study is to evaluate the neuro-ophthalmic and clinical characteristics of brain tumors in pediatric patients presenting in Macau. A retrospective case study involved 27 patients newly diagnosed with brain tumor in past 20 years at the Pediatric department of CHCSJ in Macau. Results showed vomiting (13, 48.1\%), headache (10, 37.0\%) and motor palsy $(10,37.0 \%)$ were predominant general neurological symptoms. Ocular and neuro-ophthalmic symptoms and signs presented in 16 cases, (59\% of patients). Common neuro-ophthalmic signs were ocular nerve palsy (7, 43.7\%), diplopia (6, 37.5\%) and nystagmus (5, 31.3\%). In conclusion, neuro-ophthalmic manifestation is common but easily missed in children with brain tumor. We should raise the awareness of brain tumor in pediatric patients with unexplained general symptoms such as headache and vomiting, and be aware of neurologic and neuro-ophthalmic signs.

Keywords: Pediatric; Brain Tumor; Neuro-Ophthalmic Changes

\section{Introduction}

Primary malignant central nervous system(CNS) tumors are the second most common childhood malignancies, and they are the leading cause of death from childhood cancer [1]. Common signs and symptoms of brain tumor include general neurologic symptoms such as headache, nausea and vomiting, ataxia, seizure, developmental delay, macrocephaly and endocrinopathy. However, they are either non-specific or only present at late stage. Ocular and neuro-ophthalmic manifestations may help diagnose brain tumors. These symptoms such as visual blur, diplopia, ocular nerve palsy, optic disc swelling/atrophy, ptosis, nystagmus, strabismus, proptosis, photophobia etc., can be found in a certain proportion of patients with brain tumors due to the long anatomical course of visual pathway. This study is to evaluate the neuro-ophthalmic and clinical characteristics of brain tumor in pediatric patients.

\section{Methods}

This retrospective study included 30 patients diagnosed with primary brain tumors at the Pediatric department of Centro Hospitalar Conde de São Januário in Macau in past 20 years (between January 1999 and December 2018). Medical records of each patient were reviewed, 3 cases are excluded due to lack of initial detail medical record. Therefore 27 cases were enrolled in this study. Demographic (age, sex), history of symptoms, clinical and histopathological data were recorded. All patients had clinical diagno- sis of brain tumor with confirmation by computerized tomography (CT) and/or magnetic resonance imaging (MRI). Twenty patients had histological confirmation of diagnosis.

\section{Results}

A total of 27 patients were evaluated. Ages ranged from 0 to 12 years. The mean age was $4.8 \pm 4.4$ years. Of these 27 patients, $44.4 \%(n=12)$ were in the $0-3$ years' age group, $14.8 \%(n=4)$ were in the 4-9 years' age group, and $33.3 \%(n=9)$ in 8-12 years age group (Figure 1a). $74.1 \%(n=20)$ were male and $25.9 \%(n=7)$ were female (Figure 1b).

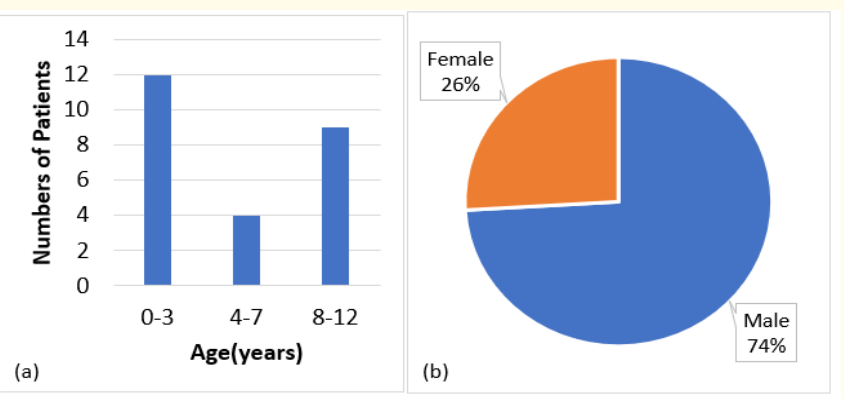

Figure 1: (a) Age group distribution of patients. (b) Gender distribution of patients. 
The patients presented with a wide variety of general and neurological symptoms (Figure 2). Among the general and neurologic symptoms, vomiting is the most common, present in $48.1 \%(n=13)$. The second are headache and motor impairment (which include muscle weakness of limbs and face), present in 37\%(n=10) each, followed by unsteady gait in $22.2 \%(\mathrm{n}=6)$, dizziness and head tilt/ turn in $14.8 \%(n=4)$ each, macrocephaly in $11.1 \%(n=3) .7 .4 \%$ $(\mathrm{n}=2)$ patients presented with anterior fontanelle bulging, diabetes insipidus and fever, and $3.7 \%(\mathrm{n}=1)$ patients presented with speak confusion, seizure and low body weight.

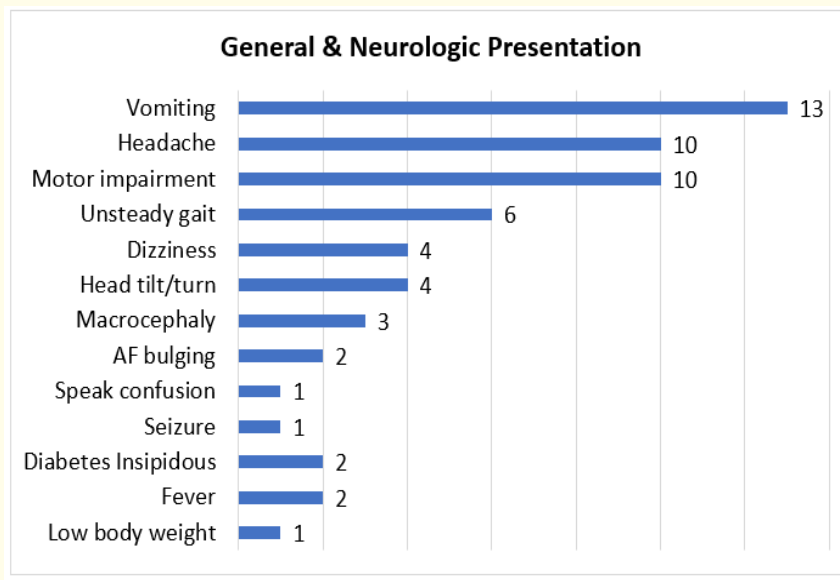

Figure 2: General and neurologic presentation.

Ocular and neuro-ophthalmic symptoms and signs presented in 16 cases, $59.3 \%$ of patients. The most common neuro-ophthalmic sign which we observed was abnormal eye movement, seen in $43.7 \%(n=7)$ of the patients, followed by diplopia in $37.5 \%(n=6)$, strabismus and nystagmus in $31.3 \%(n=5)$ patients each. Visual blur and optic disc swelling were presented in $7.4 \%(\mathrm{n}=2)$ patients each, photophobia and ptosis were seen in $3.7 \%(n=1)$ patients each (Figure 3).

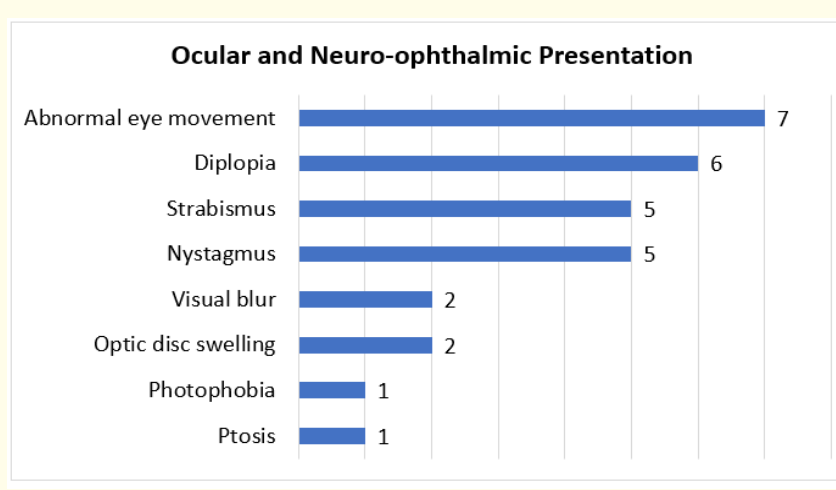

Figure 3: Ocular and Neuro-ophthalmic presentation.
The most common location of brain tumors observed in this study is posterior fossa, with a prevalence of $59.1 \%(n=16)$. The next most common location was cerebrum, pineal region and 4th ventricle, all of them account for $11.1 \%(n=3)$. There are tumors located at optic chiasm, pituitary and 3rd ventricle, with 1 case of each location respectively (Figure 4).

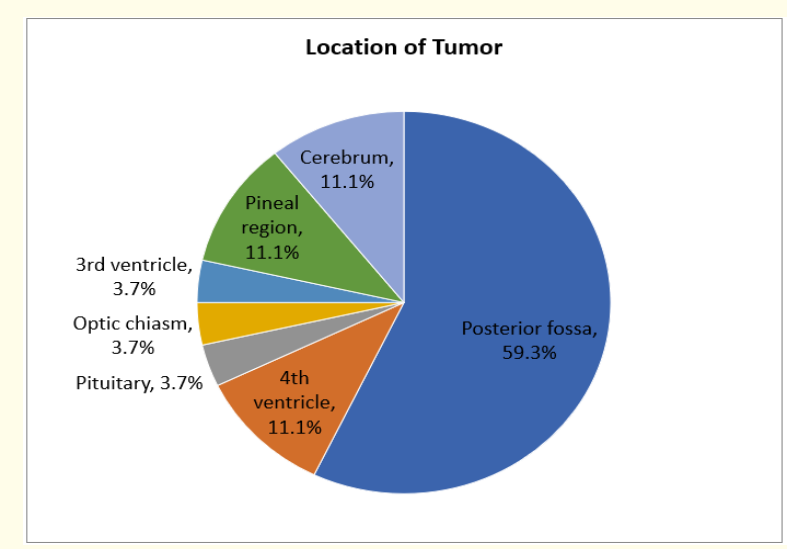

Figure 4: Location of brain tumor found in the study.

Table 1 showed the prevalence of ocular and neuro-ophthalmic presentation occurred in tumors of different location. In posterior fossa tumors, ocular and neuro-ophthalmic presentation presented in $75 \%$ of cases. In cases of tumor located at pituitary and optic chiasm, all of them have ocular presentation. Only $19 \%$ of all cases were noticed ocular presentation in the first consultation.

\begin{tabular}{|l|c|c|c|}
\hline $\begin{array}{c}\text { Location of } \\
\text { Tumor }\end{array}$ & $\begin{array}{c}\text { Case } \\
\text { count }\end{array}$ & $\begin{array}{c}\text { Case count with ocular } \\
\text { and neuro-ophthalmic } \\
\text { presentation }\end{array}$ & $\%$ \\
\hline Posterior fossa & 16 & 12 & $75.0 \%$ \\
\hline Cerebrum & 3 & 0 & $0.0 \%$ \\
\hline 4th ventricle & 3 & 1 & $33.3 \%$ \\
\hline Pineal region & 2 & 1 & $50.0 \%$ \\
\hline $\begin{array}{l}\text { Pineal region } \\
\text { + Pituitary }\end{array}$ & 1 & 1 & $100.0 \%$ \\
\hline Optic chiasm & 1 & 1 & $100.0 \%$ \\
\hline 3rd ventricle & 1 & 0 & $0.0 \%$ \\
\hline
\end{tabular}

Table 1: Prevalence of ocular and neuro-ophthalmic presentation occurred in tumors of different location.

\section{Discussion}

Primary malignant central nervous system(CNS) tumors are the second most common childhood malignancies, and they are the leading cause of death from childhood cancer [1]. Previous reports have shown that overall incidence of malignant brain and other CNS tumors varied significantly by country. In a study of global in- 
cidence of malignant brain tumors carried out by Leece, et al., there were significant differences in incidence by region. The incidence of childhood brain tumor was lowest in Southeast Africa and India, highest in the US and Canada [2]. Kimberly et al. reported that established childhood brain tumor risk factors include certain cancer syndromes and ionizing radiation exposure, and they found other environmental influences such as hazardous air pollutants, pesticides and dietary nitroso compounds are associated [3]. These may provide clues for the regional differences in incidence between developed and developing countries. However, the pattern of primary brain tumors in children was scarcely reported in East Asia, and has not been previously reported in Macau.

Ophthalmic signs and symptoms form a major part of the presentation in patients with intracranial tumors [4-7]. However, children present with less specific symptoms than adults [8], which require physicians' attention in initial examination. To our knowledge, there is a lack of relevant research about children's neuroophthalmic presentation in brain tumors, therefore this study provides further information in this field.

In this study, the children's age is range from 0 to 12 years old average is 4.8 years old, but actually there are more cases in the other two age groups, which are 0 to 3 years old and 8 to 12 years old.

Boys are significantly more than girls. Generally, females are more than males in other studies of brain tumor $[6,7]$. The greater number of females may be due to general female preponderance of meningioma's and pituitary adenomas [7], but which are not major brain tumors occur in children. In Wilne et al study about presenting features of brain tumors in children [8], he found more male cases than females.

In our series, the most common neurologic presentation is vomiting, but it is a non-specific presentation. The second are headache and motor impairment (which include muscle weakness of limbs and face). When comparing to other relevant studies, Wilne et al. [8] found that the most common symptom is headache, followed by vomiting. It may due to the average age of their study is larger than in our study ( 7.4 vs 4.8 years old). We have a majority of patients aged from 0-3 years old. At this age they are less able to express specific complaint such as headache. Wilne et al's study also found that aged 3 years or less, the first presentation, was less likely to present with headache [8].

Other neurologic presentation such as unsteady gait is also common and it is easily noticed by parents. Head tilt or turn sometimes are hard to identify, this kind of abnormal head position may indicate ocular problem such as strabismus, and it is a compensate mechanism to minimize strabismus [9]. Macrocephaly and anteri- or fontanelle bulging occur in infant. The other signs such as diabetes insipidus, low body weight and fever are subtle or non-specific.

For the neuro-ophthalmic presentation, the most common are abnormal eye movement, followed by diplopia and strabismus. In fact, these three presentations are all caused by ocular nerve palsy. The 3rd, 4th or 6th cranial nerve innervate different extraocular muscles: The oculomotor nerve (CNIII) innervates the superior rectus, inferior rectus, medial rectus and inferior oblique muscle, the trochlear nerve (CNIV) innervates the superior oblique muscle, and the abducens nerve (CNVI) innervates the lateral rectus muscle. Palsy of different nerve cause different abnormal eye movement. The nerve palsy can also cause strabismus and diplopia afterwards [10].

Since tumors' presentation depends on the tumors' location, according to the majority of ocular nerve involvement in our study, we can infer the common location of tumor is the posterior fossa, where these ocular nerves arise. As expected, in our study, posterior fossa tumors form a large proportion, about $57 \%$. The location of the brain tumor varies with age, previous study reported that in adults $70 \%$ are supratentorial, whereas in children $70 \%$ are infratentorial [11]. The location of tumors showed similar distribution in our study.

In Nisha and colleagues' study about pediatric posterior fossa tumors, abnormal ophthalmologic exam was found in initial examination in $40 \%$ of patients [12], compare to our study, ocular and neuro-ophthalmic presentation were found in a higher percentage (75\%) of posterior fossa tumors cases at diagnosis. It may due to the progression of tumor affect the ocular function at later time, or the neglect of ocular presentation in initial evaluation. In our study, ocular \& neuro-ophthalmic symptoms and signs presented in $59.3 \%$ of patients, but only $19 \%$ were noticed ocular presentation in the first consultation. Pediatricians should perform complete neurological physical examination in patients with suspected brain tumor, and ophthalmological assessment in selected cases will assist in the diagnosis.

\section{Conclusion}

Since this study is a retrospective case study, there must be some bias and limitation. To conclude, common neurologic characteristics are vomiting, headache, motor impairment and unsteady gait. Common neuro-ophthalmic characteristics are abnormal eye movement, diplopia and strabismus. Neuro-ophthalmic manifestations are common but are easily missed in children with brain tumor. We should raise the awareness of brain tumor in pediatric patients with unexplained general symptoms such as headache and vomiting, be aware of neurologic and neuro-ophthalmic signs such as abnormal eye movements, strabismus, nystagmus, abnormal pupil, ptosis and proptosis. These physical examinations are crucial and can aid in early diagnosis of brain tumors. 
Bibliography

1. Ching Lau and Wan-Yee Teo. "Overview of the management of central vervous system tumors in children" (2019).

2. Rebecca Leece., et al. "Global incidence of malignant brain and other central nervous system tumors by histology, 20032007". Neuro-Oncology 19.11 (2017): 1553-1564.

3. Johnson Kimberly J., et al. "Childhood brain tumor epidemiology: a brain tumor epidemiology consortium review". Cancer Epidemiology Biomarkers Prevention 23.12 (2014): 27162736.

4. B Leo-Kottler. "Brain Tumors Relevant to Clinical Neuro-Ophthalmology". In: B Leo-Kottler. Clinical Neuro-Ophthalmology: A Practical Guide. Berlin Heidelber: Springer-Verlag (2007): 171.

5. Kenneth W., et al. "Brain lesions with ophthalmologic manifestations". In: Kenneth W. Wright, Yi Ning J. Pediatric Ophthalmology and Strabismus. 3rd ed. New York: Oxford University Press (2012): 498-504.

6. Deshmukh S., et al. "Profile of brain tumors having ocular manifestations in a Tertiary Eye Care Institute: A retrospective study". TNOA 56 (2018): 71-75.

7. NN Tagoe., et al. "Neuro-Ophthalmic and Clinical Characteristics of Brain Tumours in a Tertiary Hospital in Ghana". Ghana Medical Journal 49 (2015): 181-186.

8. Wilne SH., et al. "The presenting features of brain tumours a review of 200 cases". Archives of Disease in Childhood 91 (2006): 502-506.

9. Kadriye Erkan Turan., et al. "Ocular Causes of Abnormal Head Position: Strabismus Clinic Data". Turkish Journal of Ophthalmology 47.4 (2017): 211-215.

10. Bekir Küçük., et al. "Amplitude of Accommodation in Patients with Multiple Sclerosis". Current Eye Research (2019): 1-7.

11. Asai A., et al. "Primary intracranial neoplasms in the first year of life". Childs Nerve System 5 (1989): 230-233.

12. Nisha Gadgil., et al. "353 Comparison of Ocular Outcomes in Pediatric Posterior Fossa Tumors”. Neurosurgery 65 (2018): 140-141.

Volume 2 Issue 12 December 2019

(C) All rights are reserved by Jorge Sales Marques., et al. 